\title{
Smoking: a major predictor of left ventricular function after occlusion of the left anterior descending coronary artery
}

\author{
W B McKENZIE, R M McCREDIE, C A McGILCHRIST, D E L WILCKEN \\ From the Department of Cardiovascular Medicine, the Prince Henry Hospital, Sydney and Department of \\ Statistics, University of New South Wales, Australia
}

SUMMARY The major predictors of left ventricular function after coronary artery occlusion were assessed in 108 consecutive patients who had complete occlusion of the left anterior descending artery as the only important lesion demonstrated at angiography between June 1978 and June 1983. A scoring system was used to identify regional damage on left ventriculograms. Forty two patients were classified as having good left ventricular function and 66 as having varying degrees of impairment. Apart from a history of myocardial infarction, the only variables discriminating between those with good and those with impaired left ventricular function were the area of distribution of the artery beyond the occlusion and cigarette smoking. Hypertension, hypercholesterolaemia, family history of vascular disease, diabetes, obesity, duration of angina, age, and presence of identifiable collaterals were not discriminators. Smoking was itself significantly associated with a history of infarction; but after controlling for this, smoking exerted a significant additional effect on the amount of left ventricular damage.

It is concluded that smoking is not only a risk factor for myocardial infarction in patients with single left anterior descending artery occlusion, but that it is also a major factor in determining the extent of associated left ventricular damage.

Disease of a single coronary artery may cause little change in ventricular function in some patients and severe impairment or aneurysm formation in others. ${ }^{1}$ It is important to identify the factors responsible for these differences, because ventricular function is the principal determinant of outcome in patients with ischaemic heart disease. ${ }^{12}$

To assess the influence of various angiographic and clinical factors on the amount of left ventricular dysfunction after occlusion of a single major coronary artery, we studied 108 consecutive patients in whom complete occlusion of the left anterior descending coronary artery was the only important luminal obstruction found at coronary angiography.

Requests for reprints to Dr R M McCredie, The Prince Henry Hospital, Little Bay, Sydney, New South Wales 2036, Australia.

Accepted for publication 20 August 1986

\section{Patients and methods}

One hundred and eight consecutive patients with isolated left anterior descending occlusion were identified from the 2150 patients who underwent coronary arteriography in our laboratory between June 1978 and June 1983. All patients had complete occlusion of the left anterior descending coronary artery, with no important ( $>50 \%$ diameter) stenoses in the circumflex or right coronary arteries. In all patients the left ventriculogram was technically adequate for assessment of regional wall motion.

The severity of left ventricular dysfunction and of coronary artery disease was assessed in each patient by the scoring system devised by Brandt $e t$ al. ${ }^{3}$ Representative systolic and diastolic frames from the $45^{\circ}$ right anterior oblique left ventriculogram were traced and the ventricular outline in diastole was divided into five segments: the anterobasal, anterolateral, apical, diaphragmatic, and posterobasal. Depending on its systolic wall motion, each segment 
was given a numerical value (normal $=0$, hypokinetic $=1$, akinetic $=2$, dyskinetic $=3$ ), and the scores of the five individual segments were summed to arrive at an overall left ventricular score. Patients with scores of 0-2 were arbitrarily classified as having "good" left ventricular function (group 1) and those with a score of 3 or more as having impaired left ventricular function (group 2).

Coronary arteriograms were performed in multiple views by the Sones' or Judkins' techniques. We used Brandt's scoring system ${ }^{3}$ to give each stenosis of a major coronary artery a numerical value that depended upon its site (proximal or distal), its severity, and its area of supply. In this system, possible coronary scores range from 0 (if all arteries are normal) to 15 (if there is a total occlusion of all three coronary arteries).

Identifiable collateral vessels were graded according to the degree of opacification of the distal left anterior descending artery seen during coronary angiography: zero if a distal vessel was not identified; 1 if the distal vessel was seen only faintly and incompletely; 2 if the distal vessel was completely opacified; and 3 if the distal segment was as well opacified as the vessel being injected. This assessment was made after both right and left coronary injections, the two values being summed to give a collateral score.

To determine the important predictors of left ventricular function after left anterior descending artery occlusion, we compared several pre-existing variables in the patients with good (group 1) or impaired (group 2) left ventricular function. These included smoking, hypertension, family history of premature vascular disease, serum cholesterol, diabetes, body mass index, and duration of angina, the latter indicating possible predisposition to collateral formation. We also assessed variables that presumably result from left anterior descending coronary artery occlusion-area of supply as reflected by coronary artery score, a history of infarction, and the presence of abnormal $Q$ waves on the electrocardiogram.

Patients were classified according to smoking history at the time of infarction or first presentation as: non-smokers, patients who had not smoked for at least two years; and light smokers, those smoking $<10$ cigarettes a day and pipe and cigar smokers; moderate smokers, between 10 and 20 cigarettes daily; heavy smokers $>20$ cigarettes a day. A period of abstention of two years was chosen for nonsmokers because data from the Framingham study, confirmed by Rosenberg et al, showed that by this time the risk of a cardiovascular event reverted to that found in people who had never smoked. ${ }^{45}$

Patients with a blood pressure $>150 \mathrm{~mm} \mathrm{Hg}$ systolic or $>95 \mathrm{~mm} \mathrm{Hg}$ diastolic or who had been treated for hypertension were regarded as having hypertension. Patients were classified as having a family history of ischaemic heart disease if ischaemic heart disease had developed in a first degree relative aged $<60$. Fasting serum cholesterol, height, weight, and body mass index were measured at the time of cardiac catheterisation.

We performed stepwise discriminant analysis (Statistical Package for the Social Sciences) of the variables shown in table 1 to discriminate between group 1 (good left ventricular function) and group 2 (impaired left ventricular function) patients. This program shows variables that discriminate between the two groups and at each step in the analysis selects the variable that gives the best discrimination. A statistic which is used to evaluate a discriminating variable is termed " $F$ to enter" and in this calculation any value $>3.96$ is significant at the $5 \%$ level. The same discriminant analysis was used when a history of infarction was included in the analysis.

\section{Results}

Of the 108 patients with isolated complete occlusion of the left anterior descending coronary artery, 90 were male (mean age 51 years, range 34-69 years) and 18 were female (mean age 51 years, range 34-63 years). Most had symptoms of angina (74), left ventricular failure (10), or arrhythmia (4) and were studied with a view to bypass surgery. Twenty were symptom free or had only slight symptoms; most of these were younger patients with a history of myocardial infarction or of atypical chest pain in whom angiography had been performed mainly as a guide to prognosis.

Ventricular function was normal (score of zero) in 22 patients and abnormal in 86. Figure 1 shows the distribution of ventricular scores among the 108 patients studied. Forty two patients had ventricular scores of 0-2 and were included in group 1 ("good" left ventricular function). The remaining 66 patients had ventricular scores of 3-9 and were included in group 2 ("impaired" left ventricular function). The cut off point of 0-2 for group 1 was chosen arbitrarily because the statistical significance of the results was not different if group 1 patients included only those with scores of $0-1$ or was extended to include those with scores of $0-3$.

Figure 2 shows the coronary artery scores in the 108 patients ranged from 3 to 9 .

Stepwise discriminant analysis of the pre-existing variables in table 1 shows that only two, smoking $(p<0.00005)$ and left anterior descending coronary score $(\mathrm{p}=0.0005)$ are significant discriminators. The program selects smoking classification as the 


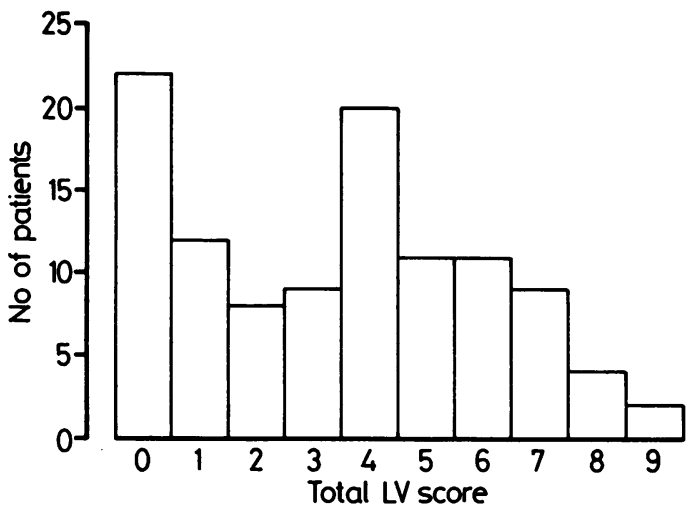

Fig 1 Distribution of left ventricular ( $L V$ ) scores determined at left ventriculography in 108 consecutive patients with isolated complete occlusion of the left anterior descending coronary artery.

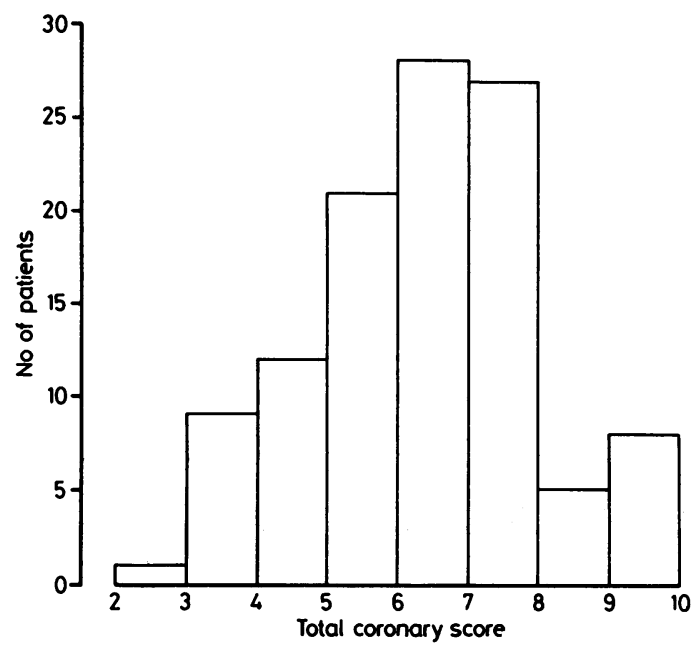

Fig 2 Distribution of coronary artery scores determined at coronary angiography in 108 consecutive patients with isolated complete occlusion of the left anterior descending coronary artery.

best discriminator. In group 1, $22(52 \%)$ of 42 were non-smokers compared with $11(17 \%)$ of 66 in group 2. After the coronary score is included the " $F$ to enter" value of smoking remains relatively unaffected. Had there been a good association between the effects of these two variables on total left ventricular score, the fitting of one would have much reduced the " $F$ to enter" of the other. There is, therefore, a largely separate effect of the two variables on left ventricular function as determined by total left ventricular score.

Table 2 shows the results when factors that presumably result from total left anterior coronary
Table 1 Stepwise discriminant analysis of pre-existing variables in 42 group 1 ("good" left ventricular function) and 66 group 2("impaired" left ventricular function) patients with isolated complete occlusion of the left anterior descending coronary artery

\begin{tabular}{lcc}
\hline & \multicolumn{2}{l}{$F$ to enter } \\
\cline { 2 - 3 } Variable & Step 1 & Step 2 \\
\hline Left anterior descending & & \\
artery score & $12 \cdot 76^{\star \star}$ & - \\
Smoking & $18 \cdot 07^{\star}$ & $16.50^{\star \star \star}$ \\
Hypertension & 0.71 & 0.81 \\
Cholesterol & 0.53 & 0.36 \\
Family history & 0.01 & 0.07 \\
Body mass index & 0.05 & 0.30 \\
Age & 1.19 & 1.79 \\
Duration of angina & 1.91 & 0.49 \\
Diabetes & 0.10 & 0.05 \\
\hline
\end{tabular}

Step 1, only smoking ( $p<0.00005)^{\star}$ and left anterior descending score $(p=0.0005)^{\star \star}$ are significant discriminators.

Step 2, inclusion of left anterior descending artery score in the analysis has little effect on the " $F$ to enter" value of smoking $(\mathrm{p}<0.0005)^{\star \star \star}$.

Table 2 Stepwise discriminant analysis between group 1 and group 2 patients by history of infarction, abnormal $Q$ waves in electrocardiograph, and collateral score

\begin{tabular}{lrll}
\hline & \multicolumn{3}{l}{ F to enter } \\
\cline { 2 - 4 } Variable & Step I & Step 2 & Step 3 \\
\hline Infarct history & $35.68^{\star}$ & - & - \\
Left anterior descending & & & \\
$\quad$ artery score & 12.76 & 15.89 & - \\
Smoking & 13.64 & 8.52 & $7.20^{\star \star}$ \\
Collateral score & 0.01 & 0.01 & 0.06 \\
Q waves & 1.18 & 1.21 & 0.91 \\
\hline
\end{tabular}

Step 1, history of infarction is the most significant discriminator in those with impaired left ventricular function $(p<0.00005)^{\star}$.

Step 2, inclusion of history of infarction and left anterior descending artery score in the analysis reduces the " $F$ to enter" value of smoking, but it still remains significant $(p=0.008)^{\star \star}$.

artery occlusion-history and electrocardiographic evidence of infarction and formation of angiographically visible collateral vessels-were included in the analysis. History of infarction is a significant discriminator $(p<0.00005)$ but not the presence of abnormal $Q$ waves in the electrocardiogram $(p=$ 0.28 ) or the formation of identifiable collateral vessels $(p=0.91)$. When history of infarction was included in the analysis, however, the " $F$ to enter" value of smoking remained highly significant $(p=$ 0.008 ); that is smoking remained an additional independent discriminator. Also discriminant analysis showed that smoking was significantly more common in those with a history of infarction ( $p=$ 0.014). 


\section{Discussion}

In this group of patients in whom complete occlusion of the left anterior descending coronary artery was the only important lesion ventricular function ranged from normal to severely impaired. The differences presumably reflect variations in the incidence and extent of myocardial infarction. Of the variables analysed in an attempt to explain these differences, apart from a history of myocardial infarction, only the area of supply distal to the left anterior descending occlusion and history of cigarette smoking were of significance in discriminating between patients with good and impaired left ventricular function. Discriminant analysis established that these two variables were acting independently. This remained true if the cut off point for group 1 ("good" ventricular function) was taken at a ventricular score of 2 or 3 , and even if the nine light smokers in this study were classified as smokers rather than non-smokers. Age, sex, family history of vascular disease, or abnormal $Q$ waves in the electrocardiogram, collateral score, or the presence of other major risk factors for coronary artery diśease did not discriminate between the two patient groups.

It is known that lesions of the left anterior descending artery at different sites are associated with differences in left ventricular function. Patients with stenoses of the proximal left anterior descending artery have greater depression of ejection fraction, ${ }^{6}$ more abnormal wall motion, ${ }^{7}$ and more profound exercise dysfunction ${ }^{8}$ than do similar patients with distal stenoses. Such findings are not unexpected because the quantity of myocardium dependent upon a major coronary artery decreases as flow progresses more distally in the vessel. The results of the present study accord with these findings.

Cigarette smoking is associated with more severe and extensive coronary atherosclerosis and smokers have a considerably increased risk of both myocardial infarction (as shown here) and cardiovascular death. This increases with the number of cigarettes smoked. ${ }^{9-11}$ In this study we also found that after occlusion of a single major coronary artery left ventricular function was more depressed in heavy smokers than in a group of light and non-smokers with similar lesions. This association is independent of the site of arterial occlusion, the size of distribution of the artery, and a history of infarction and suggests that cigarette smoking independently affects the amount of myocardial infarction that results from the occlusion of a particular artery.

Support for this suggestion comes from a study of 3997 patients undergoing coronary angiography which showed a greater frequency of previous myo- cardial infarction in smokers than in non-smokers with coronary artery disease of comparable severity. ${ }^{12}$ Left ventricular function was not assessed in that study, however, and the inclusion of a heterogeneous group of patients with multivessel disease makes separation of the effects of smoking difficult. Epidemiological studies have also shown that although smoking is strongly related to myocardial infarction and death, the relation to angina pectoris is modest, if it exists at all. ${ }^{13}$ These findings also suggest an independent effect of cigarette smoking on the likelihood and size of myocardial infarction.

Acute thrombosis of a previously chronically narrowed coronary vessel is the pathogenic mechanism responsible for most transmural myocardial infarctions $^{14}$ and cigarette smoking may promote thrombosis by increasing platelet aggregation and adherence. ${ }^{15-20}$ Cigarette smoking seems to affect the biochemical composition of atherosclerotic plaques $^{21}$ and this may influence their rates of growth and thrombogenic potential. A recent study has also shown that production of prostacyclin, a potent vasodilator and platelet antiaggregatory agent produced by vascular endothelial cells, is depressed in smokers. ${ }^{22}$

The importance of these smoking induced changes lies in their potential for altering the time course over which occlusion of a vessel takes place and consequently the amount of left ventricular damage that results. We suggest that cigarette smoking mainly affects ventricular function by promoting thrombus formation at sites of atherosclerotic narrowing, and that in smokers the development of sudden complete occlusion may be more likely than progressive gradual narrowing of non-critically narrowed vessels. The consequences for left ventricular function will be great because the time taken for a stenosis to develop has a major bearing on the development and size of collateral vessels in both animal and human studies ${ }^{2324}$ and collateral flow is a major determinant of the amount of myocardial damage occurring after coronary occlusion. ${ }^{20}$ Our finding that there was no significant difference in collateral score between patients with good and impaired left ventricular function may simply reflect the fact that coronary angiography only visualises epicardial coronary arteries with diameters $>100 \mu \mathrm{m}$. Most human collaterals are smaller than this and are subendocardial. Therefore, the number and size of collaterals identified at angiography may have only limited relevance to collateral flow. ${ }^{25}$

Changes in heart rate, blood pressure, plasma catecholamines, and carboxyhaemoglobin concentrations are also seen in smokers and these provide further potential mechanisms through which cigarette smoking may influence infarct size. Other 
variables such as autonomic tone and drug treatment at the time of infarction may also be important. Whatever the mechanisms, however, the present study identifies a clear cut association between smoking and ventricular function among a group of symptomatic and asymptomatic patients with occlusion of the left anterior descending coronary artery. Furthermore, the power of the association we found strongly suggests that the smoking habit is an important independent determinant of left ventricular function in patients with ischaemic heart disease.

We thank Mr G Milstead for help with the statistical analysis.

\section{References}

1 Bruschke AV, Proudfit WL, Sones FM. Progress study of 490 consecutive non surgical cases of coronary disease followed for 5 to 9 years. II Ventriculographic and other correlations. Circulation 1977;47:1154-63.

2 Harlan WR, Oberman A, Grimm R, Rosati RA. Chronic congestive heart failure in coronary artery disease: clinical criteria. Ann Intern Med 1977;86: $133-46$.

3 Brandt PW, Partridge JB, Wattie JW. Coronary arteriography: method of presentation of the arteriogram report and scoring system. Clin Radiol 1977;28:361-5.

4 Gordon T, Kannel WB, McGee D, Dawber TR. Death and coronary attacks in men after giving up cigarette smoking. A report from the Framingham study. Lancet 1974; ii:1345-8.

5 Rosenberg L, Kaufman DW, Helmrich SP, Shapiro S. The risk of myocardial infarction after quitting smoking in men under 55 years of age. $N$ Engl $F$ Med 1985;313:1511-4.

6 Kumpuris AG, Quinones MA, Kanon D, Miller RW. Isolated stenosis of left anterior descending or right coronary artery: relation between site of stenosis and ventricular dysfunction and therapeutic implications. Am F Cardiol 1980;46:13-20.

7 Brooks N, Cattell M, Jennings K, Balcon R, Honey M, Layton C. Isolated disease of left anterior descending coronary artery: Angiocardiographic and clinical study of 218 patients. Br Heart $\mathcal{F}$ 1982;47:71-7.

8 Leong $\mathrm{K}$, Jones RH. Influence of the location of left anterior coronary artery stenosis on left ventricular function during exercise. Circulation 1982;65: 109-14.

9 Auerbach O, Carter HW, Garfinkel LA, Culyer Hammond E. Cigarette smoking and coronary artery disease. A macroscopic and microscopic study. Chest
1976;70:697-703.

10 Doyle JT, Dawber TR, Kannel WB, Kinch SH, Khan HA. The relationship of cigarette smoking to coronary heart disease-the second report of the combined experience of Albany NY and Framingham Massachusetts studies. fAMA 1964;190:886-90.

11 Ramsdale DR, Faragher EB, Bray CL, Benett DH, Ward C, Beton DC. Smoking and coronary artery disease assessed by routine coronary arteriography. Br Med f 1985;290:197-200.

12 Hartz AJ, Barboriak PN, Anderson AJ, Hoffman RG, Barboriak JJ. Smoking, coronary artery occlusion and nonfatal myocardial infarction. $\mathcal{F} A M A$ 1981; 246:851.

13 Kannel WB. Cigarettes, coronary occlusions and myocardial infarction. $\mathcal{F} A M A$ 1981;246:871-2.

14 Rentrop P, Blanke H, Kanch KR, Kainer H, Kortering $\mathrm{H}$, Leitz K. Selective intracoronary thrombolysis in acute myocardial infarction and unstable angina pectoris. Circulation 1981;63:307-17.

15 Fuster V, Chesebro JH, Frye RL, Elveback LR. Platelet survival and the development of coronary artery disease in the young adult: effects of cigarette smoking, strong family history and medical therapy. Circulation 1981;63:546-51.

16 Mustard JF, Murphy EA. Effect of smoking on blood coagulation and platelet survival in man. Br Med $\mathcal{f}$ 1963;i:846-9.

17 Levine PH. An acute effect of cigarette smoking on platelet function. Circulation 1973;48:619-23.

18 Davis JW, Davis RF. Acute effects of tobacco cigarette smoking on the platelet aggregate ratio. $\mathrm{Am} \mathcal{F} \mathrm{Med}$ Sci 1979;278:139-43.

19 Woolf N, Pitillo RM, Machin SJ. Cigarette smoking and platelet adhesion. Lancet 1983;ii: 1091.

20 Rivas F, Cobb FR, Bache RJ, Greenfield JC. Relationship between blood flow to ischaemic regions and extent of myocardial infarction-serial measurement of blood flow to ischaemic regions in dogs. Circ Res 1976;38:439-47.

21 Ribeiro $P$, Walesby R, Edmonson S, Jadhav AV, Trayner J, Oakley CM. Collagen content of atherosclerotic arteries is higher in smokers than in nonsmokers. Lancet 1983;i:1070-3.

22 Nadler JL, Velacco JS, Horton R. Cigarette smoking inhibits prostacyclin formation. Lancet 1983;ii: 1248-50.

23 Fulton $W$. The time factor in the enlargement of anastomosis in coronary artery disease. Scott Med $\mathcal{F}$ 1964;9:18-23.

24 Flameng W, Schwarz F, Schafer W. Coronary collaterals in the canine heart-development and functional significance. Am Heart F 1979;97:70-7.

25 Newman PE. The coronary collateral circulation: determinants and functional significance in ischaemic heart disease. Am Heart $\mathcal{F}$ 1981;102:431-45. 